Musées, Patrimoine et Culture scientifiques et techniques

182 | 2019

mars-avril 2019

\title{
«Cabanomusée » : une expérience de muséographie participative à la Cité des Sciences et de l'Industrie
}

\section{Flora Ploquin}

\section{(2) OpenEdition \\ 12 Journals}

\section{Édition électronique}

URL : http://journals.openedition.org/ocim/2294

DOI : $10.4000 /$ ocim.2294

ISSN : 2108-646X

Éditeur

OCIM

\section{Édition imprimée}

Date de publication : 1 mars 2019

Pagination : 10-17

ISSN : 0994-1908

\section{Référence électronique}

Flora Ploquin, « "Cabanomusée » : une expérience de muséographie participative à la Cité des

Sciences et de l'Industrie », La Lettre de I'OCIM [En ligne], 182 | 2019, mis en ligne le 01 mars 2020, consulté le 17 mars 2020. URL : http://journals.openedition.org/ocim/2294 ; DOI : https://doi.org/ 10.4000/ocim.2294

Ce document a été généré automatiquement le 17 mars 2020

Tous droits réservés 
«Cabanomusée »: une expérience de muséographie participative à la Cité des Sciences et de l'Industrie

Flora Ploquin

Premiers schémas de conception de l'« Astérocabane »

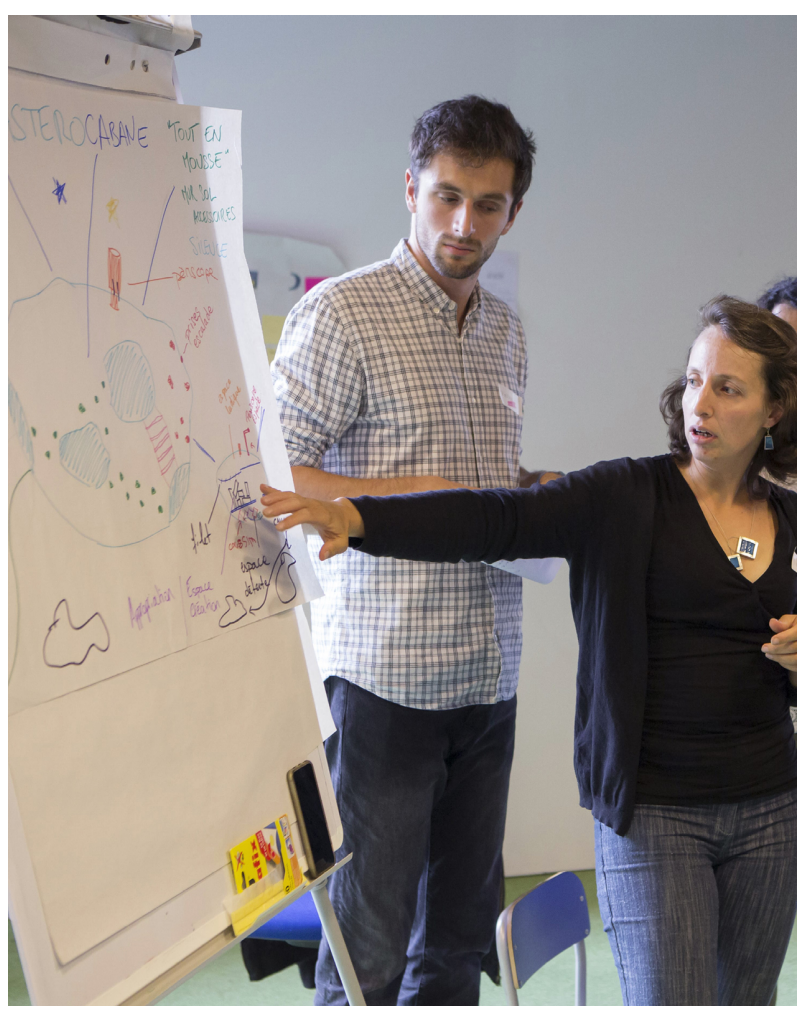

(c) EPPDSCI - Mélanie Challe 


\section{Un module d'exposition co-conçu avec le public}

1 Les cabanes inspirent à chacun d'entre nous des représentations très diverses : un drap posé sur une table, quelques branchages assemblés, un refuge de montagne, une nuit d'hôtel perchée dans les arbres ou un abri d'urgence. Tout le monde semble avoir quelque chose à raconter sur les cabanes : un souvenir, une méthode, un avis. C'est pourquoi dès le départ le sujet de l'exposition a semblé propice aux muséographes pour donner la parole à différents "experts» de la cabane : des architectes, artistes, habitants de cabanes, mais également des enfants, parents, passionnés de cabanes, ou simplement curieux.

2 C'est donc dans le cadre de cette large démarche de consultation des publics que s'inscrit le projet participatif «CabanoMusée », mené pour sa part uniquement avec des adultes ${ }^{1}$.

3 La Cité des Sciences et de l'Industrie a déjà une forte pratique d'implication des publics (comité de visiteurs, consultation du public, nombreux tests menés avec les publics en phase de conception d'exposition...), c'est néanmoins la première fois que le public est invité à co-concevoir un élément d'exposition de cette façon.

4 Effectivement, ici, bien plus que d'interroger le public sur ses attentes, l'objectif est de lui proposer de concevoir un ou plusieurs éléments muséographiques originaux pour l'exposition Cabanes: faire émerger des idées, puis les mener ensemble, dans un partage de créativité, de connaissances et de savoir-faire entre publics et professionnels. Après tout, l'activité de construction de la cabane, ne représente-t-elle pas l'archétype du faire soi-même et du faire ensemble?

5 À l'heure où les projets dits participatifs se multiplient - et notamment dans la sphère muséale - ce projet a été conçu afin de questionner une démarche en l'expérimentant : peut-on co-construire une exposition avec du public non-spécialiste de la muséographie ? Comment? Cela apporte-t-il une réelle plus-value au projet?

6 Si le fait d'associer les visiteurs à la conception d'une exposition n'est pas une idée nouvelle, les modes de participation, eux, sont en plein renouvellement. D'un côté, l'essor des outils numériques bouleverse les modes de communication entre les institutions et leurs publics, créant de nouveaux espaces de partage en ligne des savoirs. De l'autre, un besoin criant de se réunir, de mettre la main à la pâte, de faire ensemble et de faire soi-même se fait ressentir. Surfant sur la vague du Do it Yourself, les Fab Labs ${ }^{2}$ essaiment en France et à l'étranger ${ }^{3}$. Ouvert en 2014, celui de la Cité des Sciences et de l'Industrie s'accompagne d'un Living $\mathrm{Lab}^{4}$, le tout étant regroupé au sein d'un espace dédié appelé le Carrefour Numérique ${ }^{2}$. Venus d'Amérique du Nord, ces lieux partagés ont pour but d'associer les usagers en amont à la création de produits et de services, mettant à l'honneur les méthodes de l'innovation ouverte. S'inscrivant dans cette mouvance et dans la lignée d'autres expérimentations menées par exemple à la Casemate ${ }^{6}$ ou à l'Exploradome ${ }^{7}$, le projet « CabanoMusée » a rapidement été soutenu par le Carrefour Numérique ${ }^{2}$ dans le cadre de sa résidence «MuséoCamp - Le musée nouvelle génération ». 
Un projet en résidence utilisant les méthodes de l'innovation ouverte (concept : EPPDCSI, Barbara Govin et Aline Rollin ; illustration : Élizabeth Holleville et Barbara Govin)

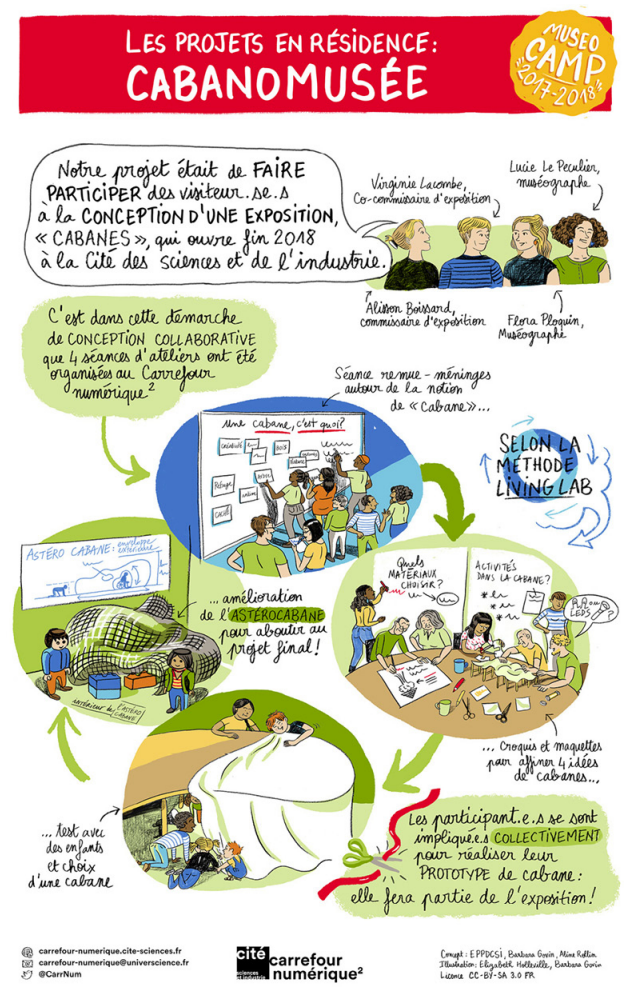

(c) Licence CC-BY-SA 3.0 FR

\section{Réunir un groupe d'utilisateurs motivés}

\section{Lancement d'un appel à participation en ligne}

7 En juin 2017, un appel à participation est publié sur Facebook et dans la newsletter d'Universcience. Celui-ci propose aux candidats de répondre à un court questionnaire, afin de connaître leurs motivations. Aucun prérequis n'est nécessaire pour participer, le but étant d'ouvrir l'expérience à la plus grande diversité de profils. Sur la quarantaine de candidatures reçues, un groupe d'une quinzaine de personnes, âgées de 23 à 71 ans, est ainsi constitué : un nombre raisonnable pour favoriser l'effervescence créative tout en laissant à chacun la possibilité d'exprimer ses points de vue et se faire entendre.

\section{Le mot d'ordre : l'expérimentation}

Quatre séances de conception se déroulent de juillet à décembre 2017. Prévues au départ sur des demi-journées, celles-ci s'avèrent rapidement trop courtes au goût des participants. À leur demande, les ateliers sont donc prolongés sur des journées entières. Ainsi fonctionne "CabanoMusée ", pas à pas, de manière expérimentale. Ce projet est avant tout vécu comme une découverte méthodologique, une expérience pour le public autant que pour les muséographes. Chaque séance est évaluée précisément par les équipes de la Cité des Sciences et de l'Industrie et par les 
participants. Ces derniers s'expriment à travers des questionnaires distribués à chaque fin de séance, mais également plus librement sur un document partagé en ligne. De cette façon, des ajustements sont faits entre chaque rencontre.

Dès le départ, le projet est présenté au groupe comme une expérimentation. Aucune attente n'est formulée en termes de résultats. Une trace de ce travail sera présentée dans l'exposition, mais dans une forme non définie à l'avance. Si, pour des raisons techniques, l'élément conçu par les participants ne peut être fabriqué, alors un compte rendu de l'expérience ainsi que les plans ou maquettes réalisés pendant les séances pourraient être exposés. Une seule certitude : l'implication des participants sera bel et bien valorisée dans l'exposition, à commencer par le générique où leurs noms apparaîtront.

\section{Première séance de créativité et de brainstorming}

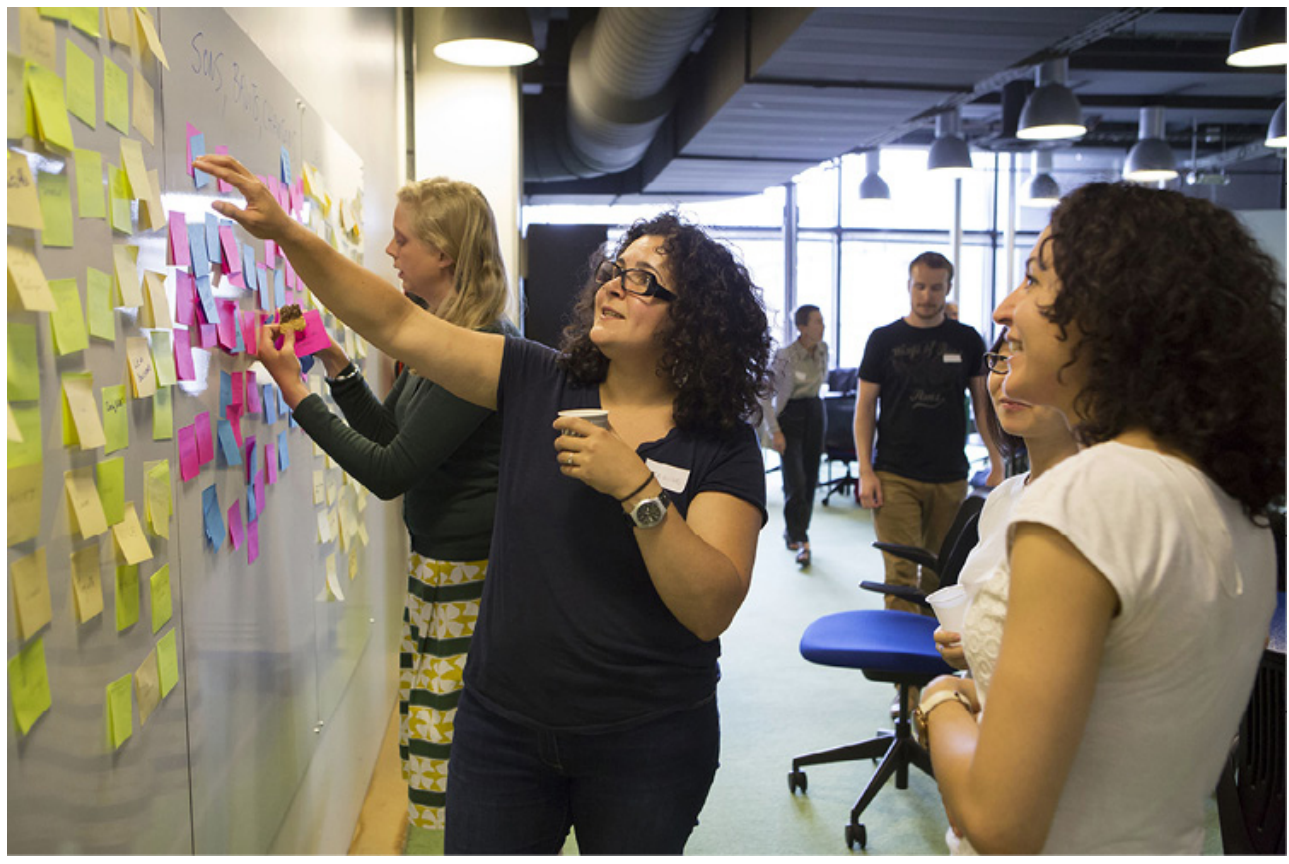

(c) EPPDSCI - Mélanie Challe

\section{Faire appel aux imaginaires du public}

Inspiré par les méthodes du design thinking ${ }^{8}$ souvent mises à l'œuvre dans les Living Lab, les rencontres sont organisées selon différentes phases : créativité et idéation, définition d'un concept, prototypage, tests, puis amélioration des premiers prototypes. Ainsi, dès la première rencontre, après avoir échangé sur la définition d'une cabane et les enjeux de la thématique, un brainstorming général est proposé sous forme de jeu créatif et les premières idées de scenarii émergent pour une cabane "originale et décalée ».

$\mathrm{Au}$ fil des séances, les idées initiales sont confrontées à plusieurs faits objectifs. Les participants sont amenés à prendre en compte toutes les contraintes de conception d'une exposition à la Cité des Sciences et de l'Industrie : sécurité des lieux et des visiteurs, accessibilité des contenus à tous, solidité et itinérance des modules. Le 
synopsis muséographique de l'ensemble de l'exposition Cabanes est également présenté. L'élément qu'ils proposeront devra s'inscrire au mieux dans l'esprit global de l'exposition : faire appel à l'imaginaire des enfants et stimuler leur créativité. Une véritable initiation au métier de muséographe est ainsi proposée autour d'une thématique originale. Les moments de travaux en groupes alternent avec des phases de mise en commun et de discussions. À l'issue du second atelier, après débats et vote argumentés, tous se mettent d'accord sur le concept qu'ils souhaitent développer : créer une "Astérocabane », un astéroïde venu de l'espace et où les enfants pourraient se réfugier.

Les premières idées sont mises sur le papier, toujours dans un esprit collaboratif

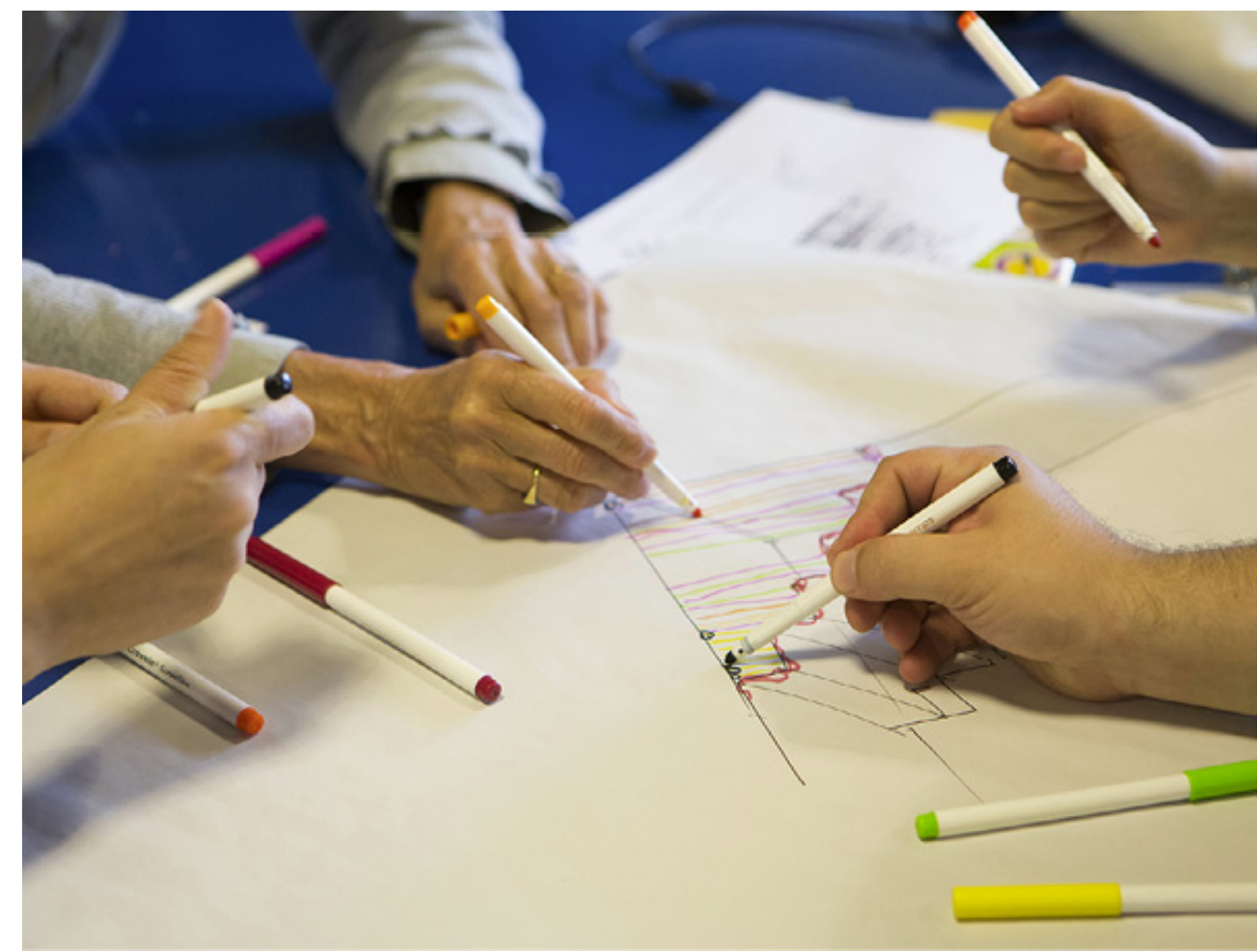

(c) EPPDSCI - Mélanie Challe

\section{Réalisation d'un module de l'exposition Cabanes}

Tous les éléments constitutifs de cette "Astérocabane " sont donc à imaginer : le scénario, les activités proposées aux futurs visiteurs, la scénographie et le design, l'ambiance lumineuse et sonore... Progressivement, les idées commencent à se matérialiser. Avec l'aide du Fab Lab de la Cité des Sciences et de l'Industrie et de ses utilisateurs, les participants prototypent leurs premières intentions. Alors que certains sont occupés à maquetter la cabane pour rendre compte de son aspect extérieur, d'autres réfléchissent à un parcours multi-sensoriel à l'intérieur de celle-ci : écoute du thérémine (ancien instrument de musique électronique inventé en 1920), observation des étoiles, toucher de différents types de surfaces ou expérimentation de la sensation d'apesanteur... 


\section{Test et amélioration des prototypes}

Rapidement, le besoin de tester les premiers prototypes avec des enfants, futurs utilisateurs de l'«Astérocabane ", se fait ressentir. Lors de la troisième séance, un échantillon de jeunes visiteurs de la Cité des enfants rejoint donc l'équipe à l'heure du goûter et donne ses impressions. Plusieurs maquettes de la cabane sont exposées aux enfants, le prototype d'un dispositif «ombre et lumière » est mis à leur portée et un parcours en hauteur autour de la notion d'espace et de vertige leur est proposé. Chacun observe comment les enfants réagissent et s'approprient ces dispositifs, puis des discussions s'en suivent.

Ces interactions avec le public-cible sont primordiales dans le processus de conception. Elles permettent de s'assurer que l'on ne part pas dans une mauvaise direction. D'ailleurs le groupe semble vraiment satisfait de l'expérience : «c'était très intéressant d'avoir des enfants qui testent. Les petits, qui ne parlent pas forcément (...) faisaient les activités sans nécessairement s'exprimer. Les plus grands se sont exprimés et nous ont dit des choses intéressantes par rapport à la forme, à ce qu'on fait à l'intérieur » explique un participant. Cela permet d'écarter certaines idées, et d'en ajuster d'autres. Concernant les jeux de lumières, «les petits ont eu tendance à vouloir trier les objets. Ils ont mis un certain temps à voir ce qu'il se passait au plafond» remarque une participante. Ce type de problématique devra donc être pris en compte par le groupe.

Maquette présentant la forme extérieure de l'« Astérocabane »

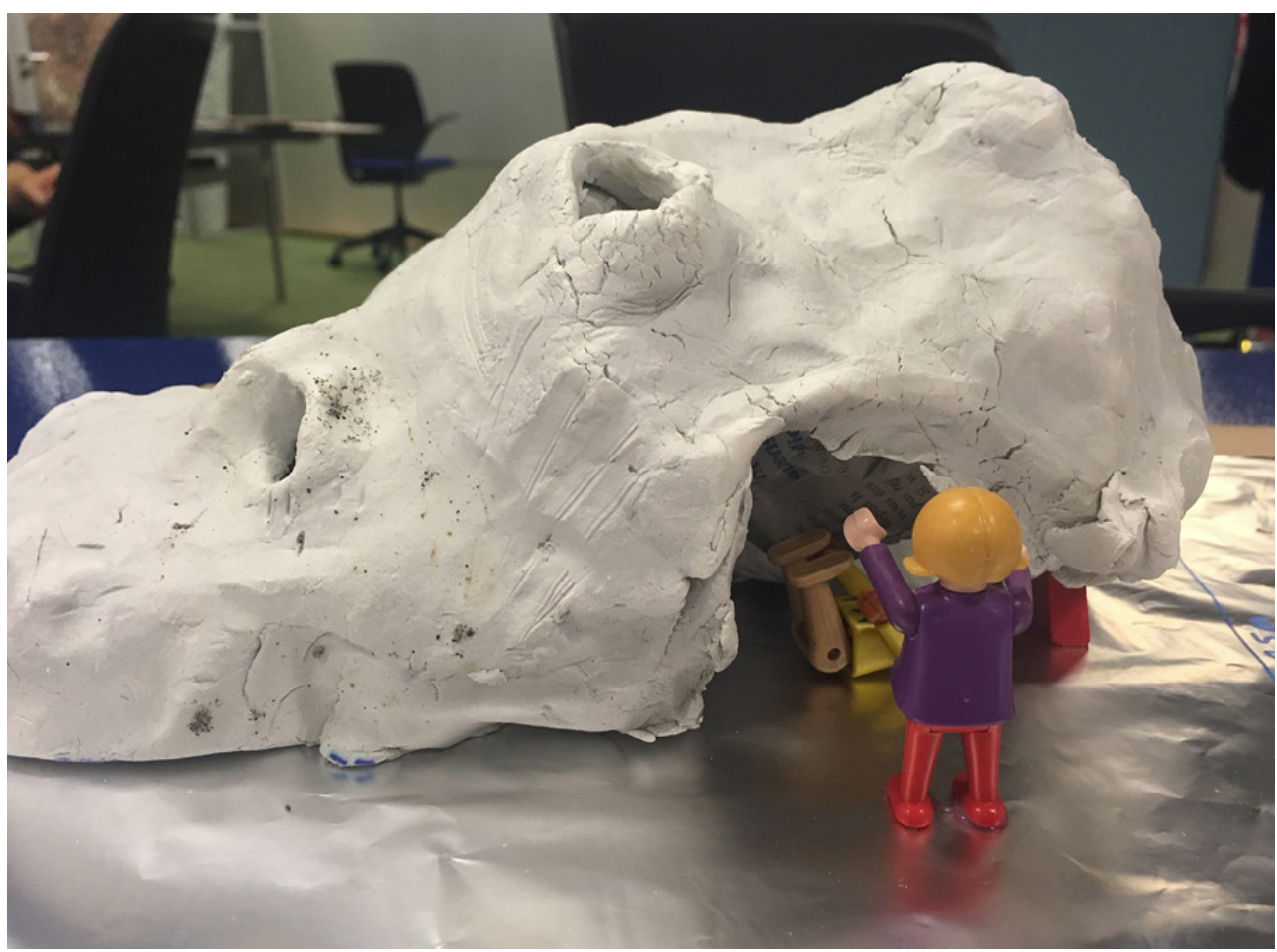

(c) EPPDSCI - Flora Ploquin 


\section{Fabrication des maquettes}

La quatrième et dernière séance est consacrée à la réalisation de maquettes et de prototypes plus avancés, qui seront présentés dans l'exposition. Au Fab Lab, certains s'initient à la découpe-laser, d'autres finalisent la conception du parcours intérieur de la cabane, tandis qu'un petit groupe sculpte la maquette de l'« Astérocabane ». Selon ce qu'ont imaginé les participants, les parois de la cabane doivent rappeler celle d'un astéroïde venu de l'espace, irrégulier et rocheux. Par opposition, l'intérieur est pensé confortable et mœlleux. L'entrée se fait par un petit tunnel, amenant les enfants à modifier leur posture ; mais une seconde entrée plus large est prévue puisque la cabane est pensée accessible aux personnes à mobilité réduite.

an tests menés avec les enfants, la manip « ombre et lumière » est transformée en un dispositif contemplatif sans pièce volante : trois postes de hauteurs différentes permettent aux jeunes visiteurs de tourner des disques afin de projeter des motifs au plafond de la cabane.

An ces quatre journées de conception, l'ensemble du groupe se montre satisfait des résultats : "le projet a vraiment pris forme!", " la séance a été très productive et a permis de faire aboutir les idées ». Certains se montrent même surpris que ces séances aient pu aboutir à des projets réalistes.

Désormais les clefs de l'«Astérocabane » sont dans les mains de l'équipe de la Cité. En phase de conception, cette cabane co-conçue est inscrite au programme de l'exposition - au même titre que les autres éléments. Lorsque les études de faisabilité pour la fabrication seront menées, l'équipe tâchera d'investir au maximum les participants dans le suivi des étapes. Pour l'heure, chacun rentre chez soi, satisfait du travail accompli et avec la promesse de se revoir en décembre 2018, pour l'inauguration.

\section{L'«Astérocabane » dans l'exposition Cabanes}

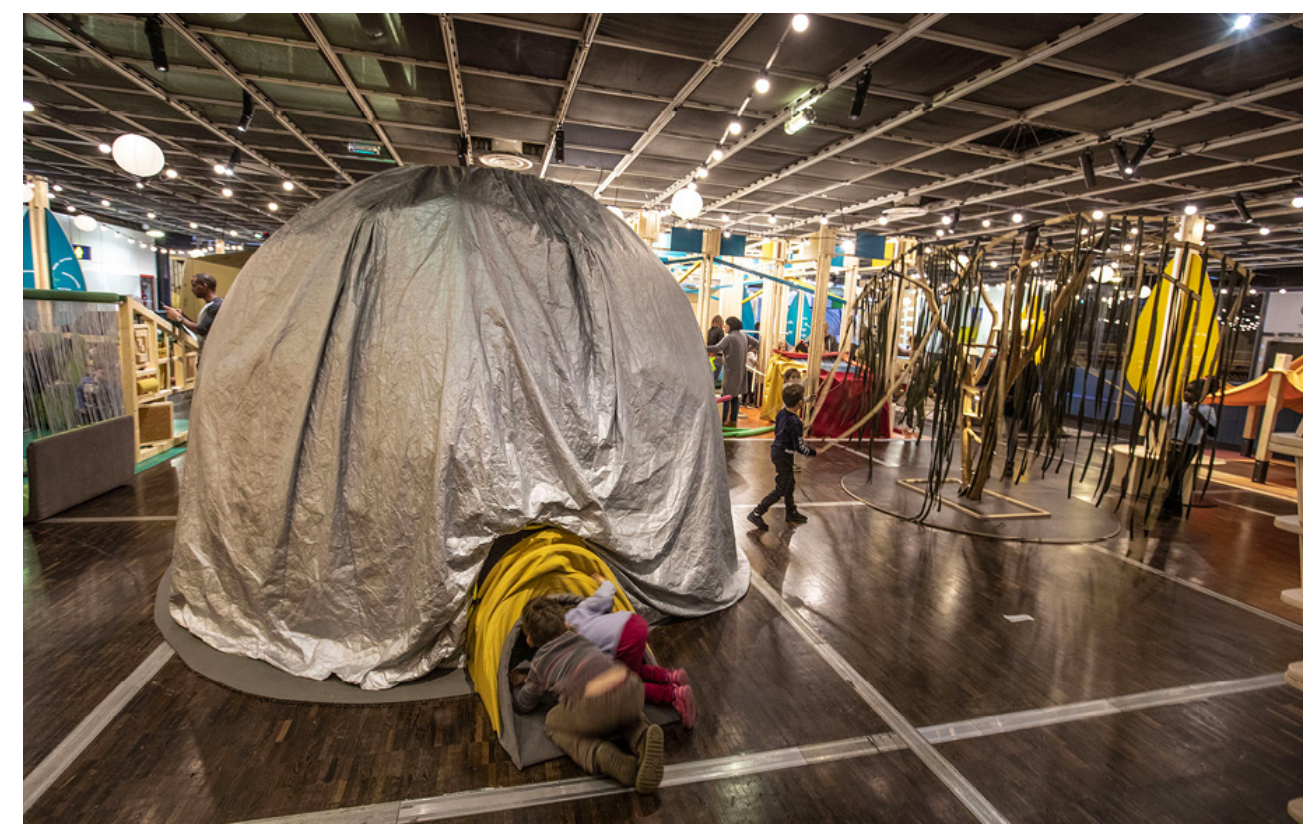

(c) EPPDCSI 


\section{Un premier bilan d'expérience}

\section{Un retour très positif des participants}

19 Tout au long du projet, les participants ont pris leur mission avec sérieux et enthousiasme. Malgré les délais entre les séances, le groupe ne s'est pas délité. Au contraire, interrogés régulièrement par écrit sur leurs ressentis, les membres du groupe déclarent avoir suivi le programme jusqu'au bout pour «le plaisir de retrouver le groupe, l'équipe, de participer à l'avancée du projet et de le voir aboutir » ou encore pour la "convivialité » et pour "l'intérêt du thème». Avec le recul, la plupart des participants expliquent que c'est la curiosité pour ce type de «démarche participative » qui les a poussés au départ à candidater, ainsi que l'envie d'entrer dans les coulisses d'une exposition et d'y œuvrer.

Bien-sûr, la muséographie ne s'apprend pas en un jour : "souvent les participants retombaient dans les mêmes erreurs que l'on fait au début de sa carrière" confie Alisson Boiffard, commissaire de l'exposition, "comme vouloir associer tout un panel d'activités dans un même module ". Effectivement, le plus dur pour le groupe a été d'effectuer des choix. De nombreuses idées ont émergé et il a fallu pousser le groupe à en sélectionner certaines et à en laisser d'autres de côté. Une vraie difficulté car il n'était pas question pour l'équipe de professionnels d'influer sur leurs propositions et de biaiser la démarche. Chaque début de séance était donc consacré à la discussion, au débat mouvant, puis à l'inévitable vote - pour ne conserver que les idées les plus unanimes. En revanche, tout a été mis en œuvre pour documenter au mieux le projet et garder une trace de toutes les propositions : grâce à l'enregistrement audio des séances, un verbatim des échanges était envoyé à tous les participants à l'issue de chaque atelier. Les séances ont été systématiquement photographiées ou filmées.

21 Du point de vue des participants, ce projet est une vraie réussite. Tous soulignent "l'ouverture, les échanges, la motivation générale et la bonne humeur » présents durant ces quelques mois de co-conception. La bonne organisation, la complémentarité des compétences sont également les principaux points forts mis en avant. Mais, à part pour les quinze participants concernés, qu'est-ce-que l'implication d'un groupe de public apporte à l'exposition elle-même ? Et à ses visiteurs?

\section{Un élément parmi les autres}

Le projet «CabanoMusée » s'est inscrit dans l'esprit de l'exposition Cabanes elle-même, qui souhaite faire la part belle à la créativité, à la co-construction et au faire ensemble. Accepter de donner la parole au public, et en l'occurrence le laisser imaginer une cabane selon ses idées et ses choix, était une volonté engagée de la part des muséographes de l'exposition dans la mesure où la "cabane " est justement l'expression de la construction sans contrainte, laissée libre à tout un chacun. Dans l'exposition, l'« Astérocabane " a donc sa place dans un environnement hétéroclite, où de nombreux univers de cabanes se côtoient.

Afin de connaître le retour des visiteurs, l'exposition Cabanes dans sa globalité fera l'objet d'une évaluation qualitative. Les visiteurs seront interrogés sur les différents modules composant l'exposition, de manière neutre, afin de repérer quel impact (ou non) aura eu l'« Astérocabane » sur leur visite. 
Destinée aux enfants de 2 à 10 ans et leurs accompagnateurs, l'exposition Cabanes a ouvert ses portes en décembre 2018. Elle présente différents univers immersifs, mais également des espaces de construction libre, permettant aux jeunes visiteurs de se saisir du matériel à leur disposition pour construire toutes sortes de cabanes à leur image.

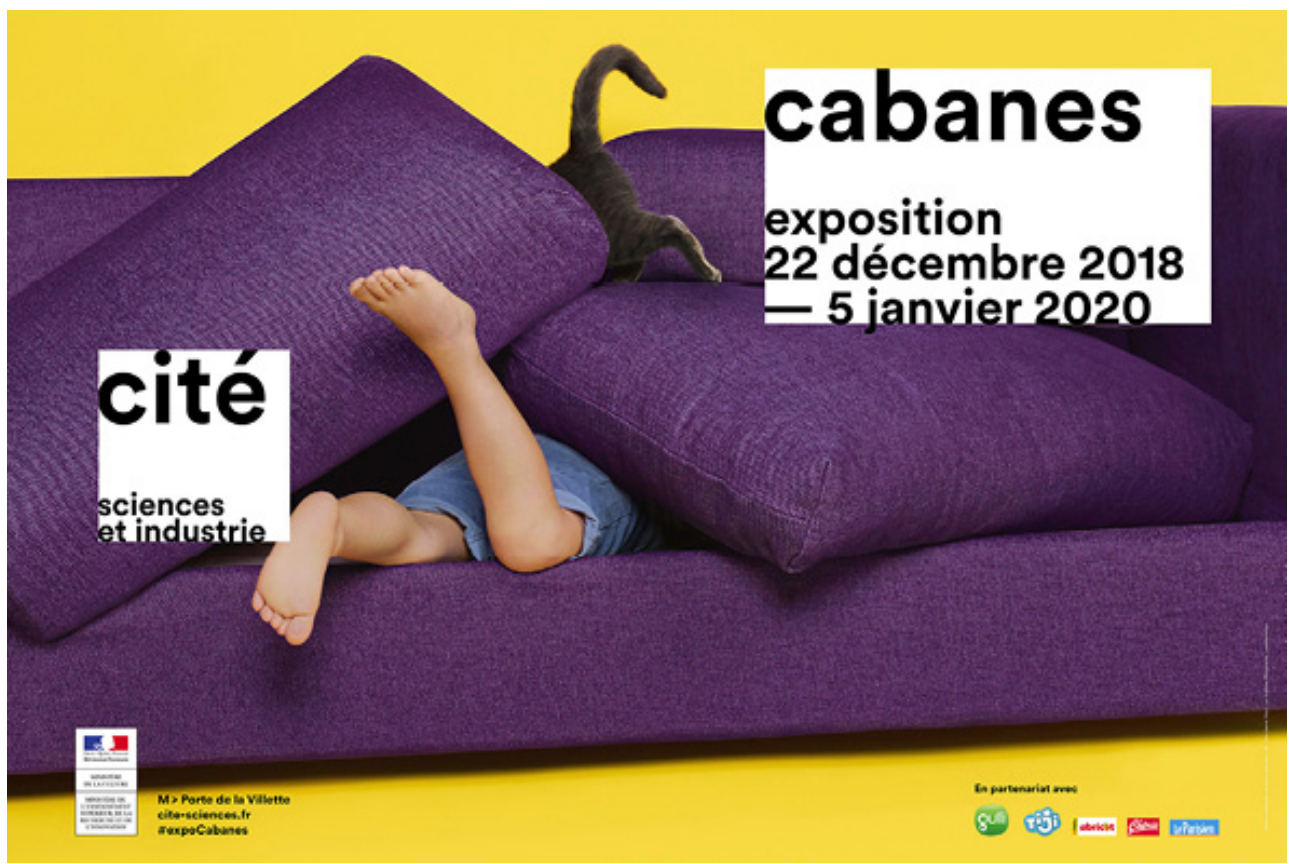

(c) EPPDCSI

\section{La co-conception, une voie pour les expositions de demain?}

Pour le muséographe, prendre en compte les représentations du public est toujours intéressant, voire primordial dans la conception d'une exposition. Cela peut se faire sous différentes formes : études préalables, consultation ou test de prototypes. Dans cette perspective, la mise en place d'une conception participative n'est qu'une étape de plus dans l'implication des publics.

D'un point de vue technique, la mise en œuvre d'une telle démarche représente un temps de travail supplémentaire incontestable pour l'équipe de muséographes. Il est à ce titre important de reconnaitre que le projet "CabanoMusée » a profité d'un renfort en ressources humaines sur plusieurs mois et de l'accompagnement très précieux des équipes du Carrefour Numérique ${ }^{2}$. De plus, mener ce type de projet requiert des compétences particulières, qui ne sont pas nécessairement celles du muséographe, comme d'animer des séances de créativité avec le public.

Ce type de projet mérite-t-il d'être mis en place pour chaque exposition ? La question reste ouverte. La façon dont ont été conçus les ateliers participatifs "CabanoMusée » ne pourrait pas s'accorder avec tous les sujets. Chaque projet d'exposition est unique et la manière de faire participer le public peut l'être également. De nombreux projets ont d'ailleurs été menés avec des objectifs et des méthodes très différentes : inviter le public à proposer des questionnements auxquels viendront répondre les contenus de 
l'exposition, ou réfléchir sur les meilleures façons de présenter ces contenus scientifiques, ou encore créer une œuvre artistique en lien avec la thématique.

Pour l'heure, la muséographie participative s'apparente ainsi plus à un patchwork d'expérimentations qu'à un mouvement général coordonné. C'est en documentant ouvertement chaque projet que cette nébuleuse participative pourra probablement être étudiée et questionnée plus en profondeur, notamment par les disciplines afférentes à la muséologie. Car, si les projets participatifs se multiplient à vue d'œil dans le secteur culturel, de nombreuses questions restent en suspens. Enjeux de démocratie culturelle, ils correspondent aussi à la politique de communication de nombreuses institutions. Si le mot participatif apparaît sur toutes les brochures, il faut bien saisir la teneur des actions qui se trouvent derrière l'expression, en se posant toujours la question : en quoi les publics ont-ils été mis à contribution? Dans quel but? Ouvrir le processus de conception au public implique donc que le sujet s'y prête, que l'équipe de professionnels adhère à cette démarche et que les modalités de mise en œuvre aient été définies en amont. Une fois ce cadre posé, le résultat de la coconception ne doit pas être prévisible (contrairement à un travail de conception classique), mais rester ouvert à tout ce que le public peut apporter à l'exposition.

\section{NOTES}

1. Un autre projet aura permis, par exemple, aux élèves de deux classes de primaire de contribuer à l'exposition en participant à la conception d'espaces de constructions libres pour les visiteurs.

2. http://carrefour-numerique.cite-sciences.fr/blog/le-jargon/\#Fab\%20Lab

3. Voir à ce sujet le numéro thématique de La Lettre de l'Ocim: Fab Labs : une (r)évolution pour la CSTI ? n¹77, mai-juin 2018.

4. http://carrefour-numerique.cite-sciences.fr/blog/le-jargon/\#Living\%20Lab

5. Voir l'article présentant le Carrefour numériue2 : Ricono, P. Devenir innov'acteur ensemble : découvrir, texte et fabriquer, La Lettre de l'Ocim, n²177, mai-juin 2018, pp. 7-12.

6. Voir l'article présentant l'exposition Confidences d'outre-tombe : Maggioni, L. Le modèle du numérique, une voie nouvelle pour mettre en scène les savoirs ? La Lettre de l'Ocim, $\mathrm{n}^{\circ} 165$, maijuin 2016, pp. 12-18.

7. www.amcsti.fr/fr/bulletin/qsec\%C2\%B2-expositions-collaboratives/

8. www.lescahiersdelinnovation.com/2016/02/qu-est-ce-que-le-design-thinking/ 


\section{RÉSUMÉS}

Au printemps 2017, une équipe de muséographes de la Cité des Sciences et de l'Industrie a souhaité faire participer un groupe de visiteurs à la conception d'éléments muséographiques d'une exposition pour enfants sur le thème des cabanes. L'une des responsables de ce projet décrit les principales étapes de cette opération, en dresse le bilan et s'interroge sur l'avenir pour les institutions muséales d'une démarche qui mêle enjeu de démocratie culturelle et politique de communication.

\section{INDEX}

Mots-clés : Public, enfants, participatif

\section{AUTEUR}

\section{FLORA PLOQUIN}

Actuellement muséographe au Muséum national d'Histoire naturelle, chargée en 2017 du projet « CabanoMusée » dans l'équipe de muséographes de la Cité des Sciences et de l'Industrie

flora.ploquin@mnhn.fr 